\title{
BMJ Open Traumatic brain injury in England and Wales: prospective audit of epidemiology, complications and standardised mortality
}

\author{
T Lawrence, ${ }^{1}$ A Helmy, ${ }^{2,3}$ O Bouamra, ${ }^{1}$ M Woodford, ${ }^{1}$ F Lecky, ${ }^{4} \mathrm{P}$ J Hutchinson ${ }^{2,3}$
}

To cite: Lawrence $T$, Helmy A, Bouamra 0, et al. Traumatic brain injury in England and Wales: prospective audit of epidemiology, complications and standardised mortality. BMJ Open 2016;6:e012197. doi:10.1136/bmjopen-2016012197

- Prepublication history and additional material is available. To view please visit the journal (http://dx.doi.org/ 10.1136/bmjopen-2016012197).

$\mathrm{TL}$ and $\mathrm{AH}$ are joint first authors.

Received 7 April 2016 Revised 22 July 2016 Accepted 25 July 2016

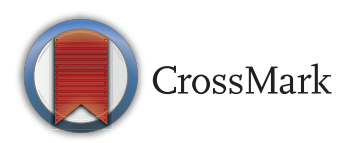

For numbered affiliations see end of article.

Correspondence to

Dr A Helmy;

adelhelmy@cantab.net

\section{ABSTRACT}

Objectives: To provide a comprehensive assessment of the management of traumatic brain injury (TBI) relating to epidemiology, complications and standardised mortality across specialist units.

Design: The Trauma Audit and Research Network collects data prospectively on patients suffering trauma across England and Wales. We analysed all data collected on patients with TBI between April 2014 and June 2015.

Setting: Data were collected on patients presenting to emergency departments across 187 hospitals including 26 with specialist neurosurgical services, incorporating factors previously identified in the Ps14 multivariate logistic regression (Ps14 ${ }^{\mathrm{n}}$ ) model multivariate TBI outcome prediction model. The frequency and timing of secondary transfer to neurosurgical centres was assessed.

Results: We identified 15820 patients with TBI presenting to neurosurgical centres directly (6258), transferred from a district hospital to a neurosurgical centre (3682) and remaining in a district general hospital (5880). The commonest mechanisms of injury were falls in the elderly and road traffic collisions in the young, which were more likely to present in coma. In severe TBI (Glasgow Coma Score (GCS) $\leq 8$ ), the median time from admission to imaging with CT scan is 0.5 hours. Median time to craniotomy from admission is 2.6 hours and median time to intracranial pressure monitoring is 3 hours. The most frequently documented complication of severe TBI is bronchopneumonia in 5\% of patients. Risk-adjusted W scores derived from the Ps $14^{\mathrm{n}}$ model indicate that no neurosurgical unit fell outside the 3 SD limits on a funnel plot.

Conclusions: We provide the first comprehensive report of the management of TBI in England and Wales, including data from all neurosurgical units. These data provide transparency and suggests equity of access to high-quality TBI management provided in England and Wales.

\section{INTRODUCTION}

Traumatic brain injury (TBI) is a major cause of mortality and morbidity. In England

\section{Strengths and limitations of this study}

- The use of registry data from all specialist units and a large number of hospitals allows a comprehensive assessment of the management of traumatic brain injury in England and Wales.

- Data from a large number of patients provides robust statistical analyses.

- Data are limited by the prespecified categories within the Trauma Audit and Research Network (TARN) data set.

- Key parameters such as Glasgow Coma Score (GCS) are collected at a single time point at admission that may not reflect the complexity of confounding factors such as resuscitation state.

and Wales, $\sim 1.4$ million patients per year attend hospital following head injury and it is the most common cause of death under the age of 40 years. ${ }^{1}$ Over the past 30 years, advances in management including the introduction of Advanced Trauma Life Support ${ }^{2}$ National Institute for Health and Care Excellence (NICE) guidelines ${ }^{1}$ and protocol-driven therapy have improved outcome $^{3}$ and reduced mortality. ${ }^{4}$ Recently, Regional Trauma Networks have been implemented in England and Wales. It is recognised, however, that major gains are still needed in terms of increasing our understanding of the pathophysiology of this heterogeneous condition and defining and optimising individual treatment strategies. The largest existing TBI data sets in the literature are from the $\mathrm{CRASH}^{5}$ study, $\sim 10000$ patients within a randomised control study of corticosteroids, and IMPACT ${ }^{6}$ a collated data set of $\sim 9800$ patients from eight randomised and three observational studies.

The UK national neurosurgical society, the Society of British Neurological Surgeons (SBNS), has established the Neurosurgical National Audit Programme (NNAP) ${ }^{7}$ the first 
comprehensive national audit of emergency and elective activity in an acute surgical specialty with a complex case mix, as a mechanism for driving quality improvement and maintaining high standards of clinical governance. Hospital and consultant surgeon level data have been collected and the first year of data relating to elective activity was published on 1 December 2014. The management of TBI differs from other aspects of neurosurgical care, in that it is heavily reliant on multidisciplinary care, including emergency medicine, neurointensive care, neurosurgery and rehabilitation medicine. In this way, surgeon-specific data are less useful and the aggregate performance of a whole unit is more indicative of the quality of care that is delivered. For this reason, the SBNS and the Trauma Audit and Research Network (TARN) have collaborated in order to produce detailed data on the management of several aspects of TBI management across England and Wales in over 15000 patients.

The objective of this study was to undertake an audit of TBI in England and Wales during a 15-month epoch (April 2014 to June 2015) specifically to define the demography, mechanism of injury, arrival mode, to characterise transfers and direct admissions to neurosurgical units, length of stay, self-reported complications and outcome in terms of mortality. We specifically sought to ascertain compliance with NICE guidance and variation in mortality according to neurosurgical centre.

\section{MATERIALS AND METHODS}

The information shown in this report is derived from the TARN registry, a prospective, observational registry of hospitalised major trauma patients in England and Wales. TARN has Health Research Authority (PIAGG Section 20) approval to conduct research on anonymised data. There was no patient involvement in the design or implementation of the study other than the oversight presented by the patient and public representatives on the TARN Board. Patients of all ages are eligible for entry to the TARN database if they suffer injuries leading to a hospital stay resulting in any of: 3 or more days in hospital, admission to intensive or high dependency care, interhospital transfer or death from injury. Patients aged over 65 years with an isolated neck of femur fracture or those with isolated closed limb fractures are excluded. Those who died at the incident scene and were not transported to hospital are not eligible. Currently the TARN database contains information on over 69000 eligible major trauma patients admitted to hospitals in England and Wales over the period of the study (April 2014 to June 2015). Each patient's injuries are centrally coded and scored reproducibly by TARN coordination centre staff using the Abbreviated Injury Scale (AIS) Dictionary ${ }^{8}$ - each injury attracts a threat to life severity code between 1 (minimal) and 6 (maximal/incompatible with life). Of these 15820 suffered a TBI (defined as an AIS 3 or greater injury to the head). Severe TBI was defined as an initial (ie, at the time the patient was admitted and assessed in the emergency department (ED)) Glasgow Coma Score (GCS) of 8 or less in combination with an AIS 3 or greater traumatic brain injury, moderate and mild TBI were defined as GCS 9-13 and $14-15$, respectively. GCS is a composite score incorporating three categorical variables: best eye opening (E), verbal $(\mathrm{V})$ and motor $(\mathrm{M})$ scores and is, de facto, the most widely used stratification metric for patients with TBI. Simple cross-tabulations and percentages were used to describe the study demography, injury mechanisms and features of the care pathway (endotracheal intubation, imaging with CT scan, transfer to a neurosurgical centre, surgical interventions and in-hospital complications) by severity of TBI for the whole study sample. Bias was avoided by collecting data on all available patients.

The following analysis focuses on these 15820 patients. Some analyses use subsets of this cohort; patients admitted directly from the scene of injury and those admitted to a neurosurgical centre. As a result of relatively small numbers of patients treated exclusively at sites without neurosurgery, these are not included in the risk-adjusted outcome analysis, this group is further filtered to only include patients whose outcome is recorded on the TARN database.

Outcome, measured as mortality is considered by using a derivation of the Ps14 multivariate logistic regres$\operatorname{sion}^{9}\left(\operatorname{Ps} 14^{\mathrm{n}}\right)$ model. The Ps $14^{\mathrm{n}}$ model calculates a probability of survival for each patient based on their age, gender, initial GCS, Injury Severity Score (ISS) and any pre-existing medical conditions. The Ps $14^{\mathrm{n}}$ model adds pupillary reactivity due to its prognostic importance in head injury. ${ }^{6} 10$ The Ps $14^{\mathrm{n}}$ model was generated using 33715 cases admitted between 2010 and 2013 (inclusive) following head injury. Missing GCS and pupil reactivity values were imputed and patients with missing pre-existing medical condition data were categorised as such. The model was internally validated using bootstrap simulation. Details of the model, including coefficients and calibration information can be found in the online supplementary information.

In order to compare mortality across different centres, the predicted survival rate at 30 days or discharge (whichever is earliest), derived from the probability of survival values assigned to patients admitted to a given institution is subtracted from the observed survival rate at 30 days or discharge to generate a 'W' score. This is then risk standardised (Ws) to allow direct comparison between units by compensating for variations in admission patterns. ${ }^{11}$ A positive Ws score, therefore, indicates a better than expected rate of survival. The same outcome assessment for mortality, that is, discharge or 30-day mortality, whichever is earliest, is used in the data within the online supplementary data.

\section{RESULTS}

Figure 1 provides a summary flow chart of the numbers of patients in each cohort of the study audit, namely: 


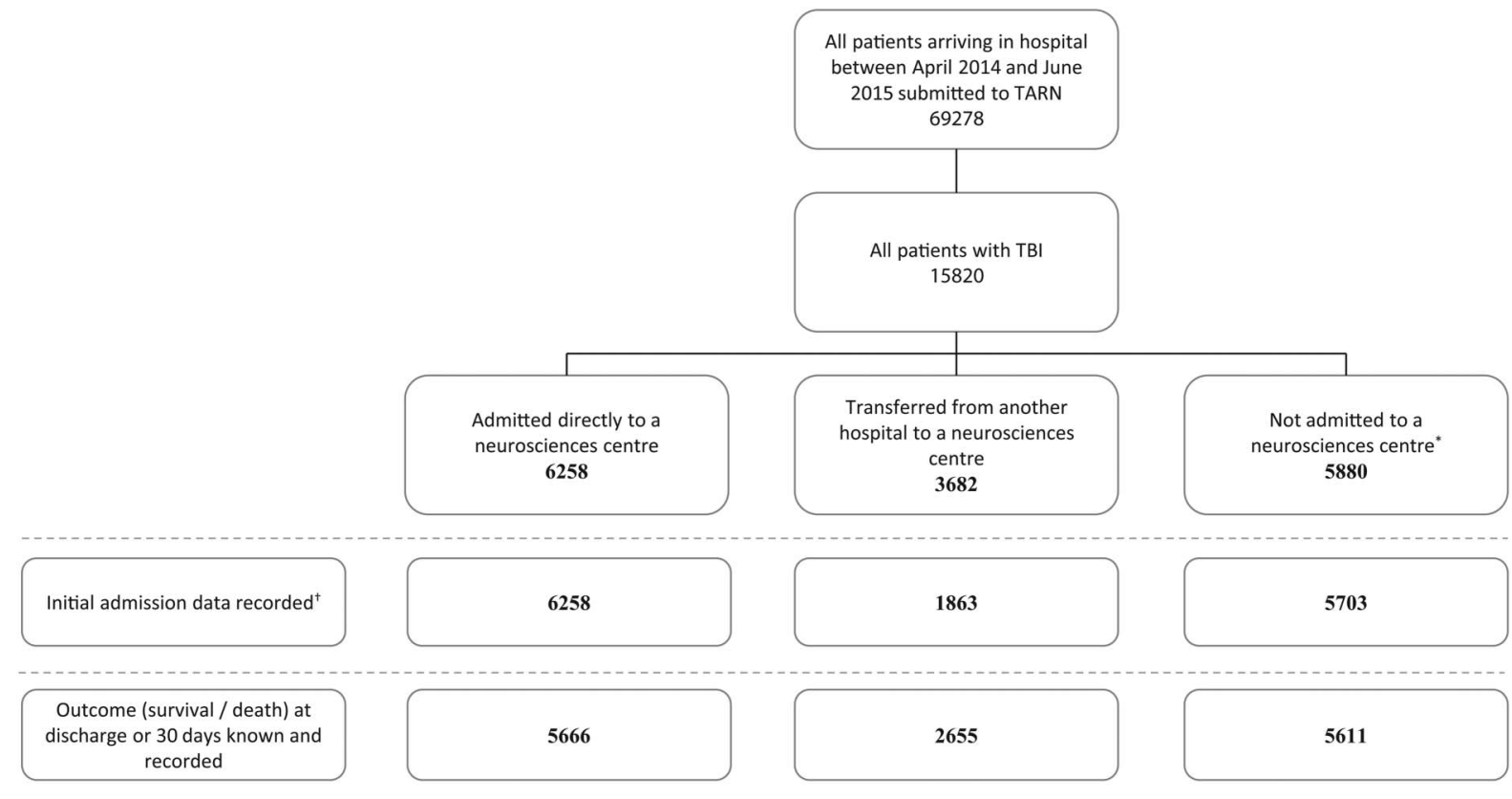

* Patients initially admitted to and treated entirely at hospitals without neuroscience care

+ Data from the scene of injury and initial hospital admitted to is recorded on TARN

Figure 1 Flow chart delineating the derivation of the TBI cohort studied. TARN, Trauma Audit and Research Network; TBI, traumatic brain injury.

those admitted directly to a neurosurgical centre $(\mathrm{n}=6358)$, those with a secondary admission (via a district general hospital, $\mathrm{n}=3682$ ) to a neurosurgical centre and those not admitted to a neurosurgical centre $(\mathrm{n}=5880)$.

\section{Demographics and mechanism of injury}

For all TBI severities, there is a unimodal age distribution with a peak in those aged between 80 and 90, and this cohort represents more than one in five of those recorded as suffering from a TBI (figure 2). For those with severe TBI, there is a smaller peak between age 20 and 30 representing just over $15 \%$ of cases (figure 3). Younger patients are more likely to be injured as a result of road traffic collisions and assaults, while with increasing age, there is a concurrent increase in the proportion of patients injured following falls under $2 \mathrm{~m}$. Of those patients with a documented admission GCS $(\mathrm{n}=15080)$, the cohort is dominated by mild TBI $(68 \%)$, with $26 \%$ with a severe TBI and only $6 \%$ with moderate TBI (table 1).

\section{Transfer to hospital and airway management}

Table 1 summarises data on hospital transfers and airway management stratified by severity of injury of TBI. The most common mode of transport to hospital is ambulance $(74 \%$ overall). Seven per cent of patients are recorded as being transported by helicopter, although in patients with severe TBI, this increases to $19 \%$. Direct admission to a neurosurgical centre from the scene of

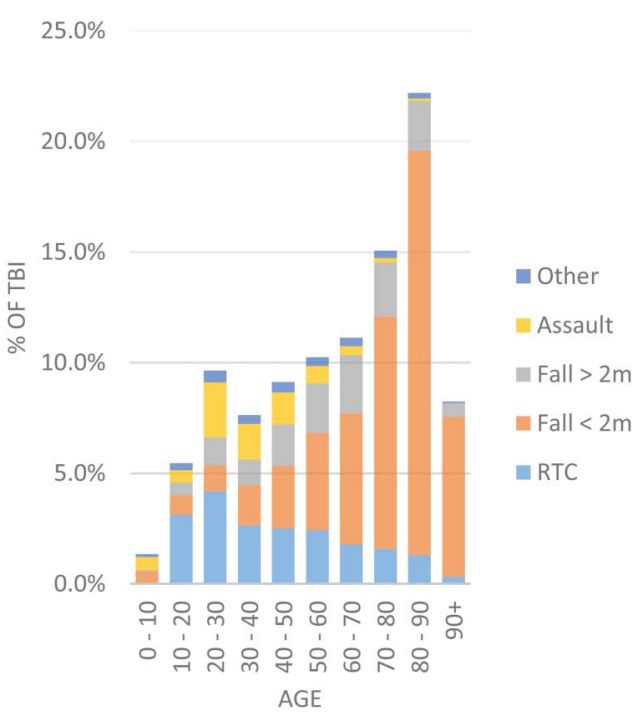

Figure 2 Proportion of all patients with TBI by age and mechanism of injury. RTI, road traffic collision; TBI, traumatic brain injury.

injury occurred in $\sim 40 \%$ of patients overall and over $60 \%$ of patients with severe TBI. This proportion was lower for patients with moderate and mild TBI $(41 \%$ and $33 \%$ ) but significant proportions were subsequently transferred (20\% and 22\%, respectively).

Over $80 \%$ of patients with severe TBI were admitted to a neurosurgical centre within 12 hours of injury with $68 \%$ within 4 hours. Eighty-six per cent of patients 
presenting with a severe TBI had definitive airway management (defined as endotracheal intubation, tracheostomy or cricothyroidotomy) prehospital or in the ED. Definitive airway management was rarely required for patients with less severe injuries.

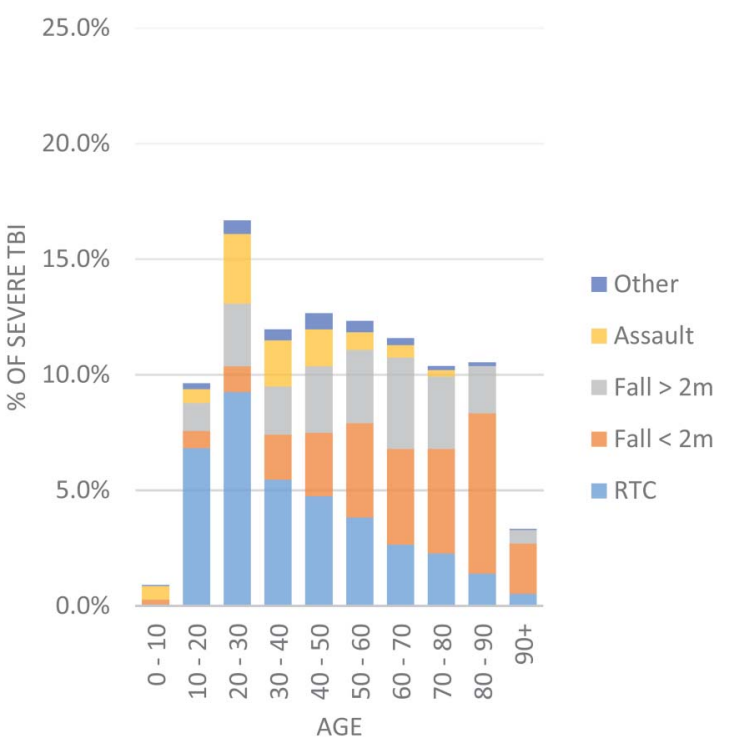

Figure 3 Proportion of patients with severe TBI (TBI in combination with $\mathrm{GCS} \leq 8$ ) by age and mechanism of injury. $\mathrm{RTI}$, road traffic collision.

\section{Time to intervention}

Table 2 summarises the data on the time intervals from injury and admission to investigation and intervention. In those patients admitted directly from the scene of injury with a GCS $\leq 8$, a median of 0.5 hours was taken to image with CT scan. Median time from arrival to imaging was 0.9 hours for moderate TBI and 1.7 hours for mild injuries. The median time taken from admission to craniotomy was 2.6 hours for severe TBI and 8.6 hours for moderate TBI. If the time to craniotomy, in severe TBI, is calculated from the time of the incident, this increases to 4 hours for direct transfers to a neurosurgical centre and 7.3 hours for those who required a secondary transfer. Median time to intracranial pressure (ICP) monitoring following admission to a neurosurgical centre was 3.1 hours following severe TBI. Smaller numbers of patients with mild or moderate TBI required craniotomy $(3.1 \%$ and $2.7 \%$, respectively) or ICP monitoring $(0.7 \%$ and $2.1 \%$, respectively), and in general, this was performed within 24 hours of arriving in hospital.

\section{Complications in hospital}

Table 3 summarises the documented complications following TBI. Overall, over 19\% of patients are recorded as suffering a complication, and in the severe TBI cohort, this incidence increases to almost $30 \%$. There is a wide range of complications; the most frequent in the

Table 1 Hospital transfer, airway management and length of stay

\begin{tabular}{|c|c|c|c|c|c|c|}
\hline Category & Group & $\begin{array}{l}\text { Severe TBI } \\
\text { n (\%) }\end{array}$ & $\begin{array}{l}\text { Moderate } \\
\text { TBI } \\
\text { n (\%) }\end{array}$ & $\begin{array}{l}\text { Mild TBI } \\
\text { n (\%) }\end{array}$ & $\begin{array}{l}\text { GCS not } \\
\text { recorded } \\
n(\%)\end{array}$ & $\begin{array}{l}\text { Total } \\
\text { n (\%) }\end{array}$ \\
\hline Mode of arrival & Ambulance & $2504(71.6 \%)$ & $662(83.8 \%)$ & $6951(76.6 \%)$ & $51(11.1 \%)$ & $10168(73.5 \%)$ \\
\hline \multirow{2}{*}{$\begin{array}{l}\text { (direct admissions, } \\
n=13 \text { 824) }\end{array}$} & Car & $3(0.1 \%)$ & $1(0.1 \%)$ & $65(0.7 \%)$ & $7(1.5 \%)$ & $76(0.5 \%)$ \\
\hline & Helicopter & $660(18.9 \%)$ & $27(3.4 \%)$ & 309 (3.4\%) & $0(0 \%)$ & $996(7.2 \%)$ \\
\hline \multirow{3}{*}{$\begin{array}{l}\text { Transfer status (all } \\
\text { patients), } \\
n=15820\end{array}$} & $\begin{array}{l}\text { Direct admission to } \\
\text { neurocentre }\end{array}$ & $2353(60.1 \%)$ & 365 (40.6\%) & 3374 (32.9\%) & $167(22.5 \%)$ & $6259(39.6 \%)$ \\
\hline & Transfer into neurocentre & $945(24.1 \%)$ & $181(20.1 \%)$ & $2298(22.4 \%)$ & $259(34.9 \%)$ & $3683(23.3 \%)$ \\
\hline & No neurocentre visit & $617(15.8 \%)$ & $353(39.3 \%)$ & $4594(44.7 \%)$ & $316(42.6 \%)$ & $5880(37.2 \%)$ \\
\hline \multirow{3}{*}{$\begin{array}{l}\text { Hours to arrival at } \\
\text { neurocentre } \\
(n=9940)\end{array}$} & $0-4$ & $2225(67.5 \%)$ & $303(55.5 \%)$ & $2621(46.2 \%)$ & $46(10.8 \%)$ & $5195(52.3 \%)$ \\
\hline & $72+$ & $23(0.7 \%)$ & $13(2.4 \%)$ & $249(4.4 \%)$ & $16(3.8 \%)$ & $301(3 \%)$ \\
\hline & Unknown & $520(15.8 \%)$ & $135(24.7 \%)$ & $1597(28.2 \%)$ & $330(77.5 \%)$ & $2582(26 \%)$ \\
\hline \multicolumn{2}{|c|}{ Median length of stay (days) (IQR) $n=15820$} & $12(3-33)$ & $11(5-24)$ & $9(5-18)$ & $10(5-21)$ & $9(4-21)$ \\
\hline Intubation location & Prehospital & $765(21.9 \%)$ & $0(0 \%)$ & $0(0 \%)$ & $0(0 \%)$ & $765(5.5 \%)$ \\
\hline \multirow{4}{*}{$\begin{array}{l}\text { (direct admissions, } \\
n=13 \text { 824) }\end{array}$} & ED & $2236(63.9 \%)$ & $0(0 \%)$ & $0(0 \%)$ & $0(0 \%)$ & $2236(16.2 \%)$ \\
\hline & Critical care & $142(4.1 \%)$ & $71(9 \%)$ & $257(2.8 \%)$ & $21(4.6 \%)$ & $491(3.6 \%)$ \\
\hline & Ward & $0(0 \%)$ & $0(0 \%)$ & $4(0 \%)$ & $2(0.4 \%)$ & $6(0 \%)$ \\
\hline & Not intubated & $354(10.1 \%)$ & $719(91 \%)$ & $8815(97.1 \%)$ & $438(95 \%)$ & $10326(74.7 \%)$ \\
\hline
\end{tabular}


Table 2 Median time to CT scanning, craniotomy and ICP monitoring from hospital arrival/incident*

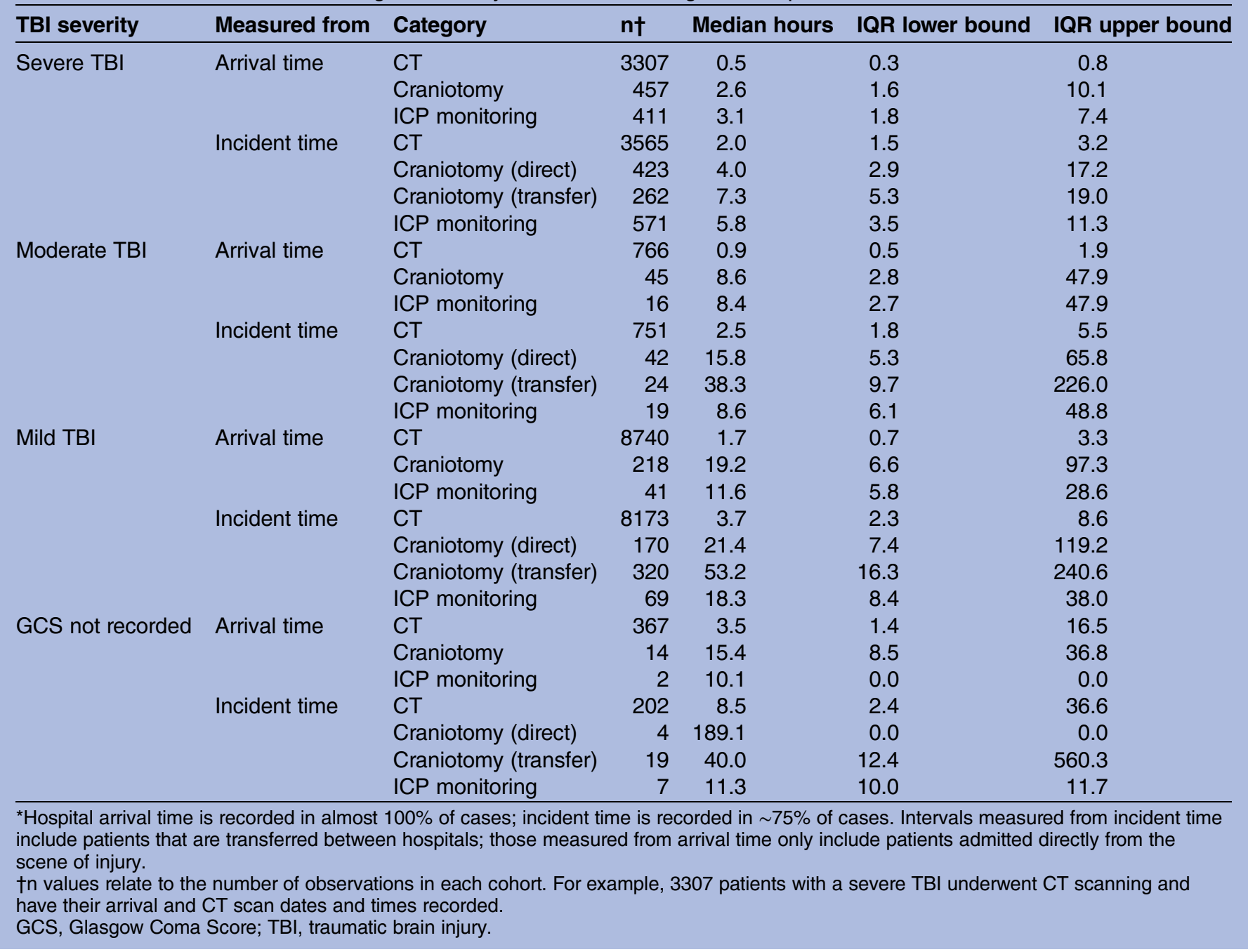

Table 3 Inpatient complications stratified by severity of injury

\begin{tabular}{|c|c|c|c|c|c|}
\hline Complication & Severe TBI & Moderate TBI & Mild TBI & GCS not recorded & Total \\
\hline Aspiration & $63(1.5 \%)$ & $8(0.9 \%)$ & $48(0.5 \%)$ & $5(0.6 \%)$ & $124(0.8 \%)$ \\
\hline Bronchopneumonia & $203(4.9 \%)$ & 32 (3.4\%) & $209(2 \%)$ & $17(2.2 \%)$ & $461(2.8 \%)$ \\
\hline Pleural effusion & $84(2 \%)$ & $9(1 \%)$ & $68(0.7 \%)$ & $12(1.6 \%)$ & $173(1.1 \%)$ \\
\hline Seizure in hospital & $119(2.9 \%)$ & $22(2.4 \%)$ & $141(1.4 \%)$ & 15 (1.9\%) & 297 (1.8\%) \\
\hline Sepsis & $129(3.1 \%)$ & $9(1 \%)$ & $107(1 \%)$ & $13(1.7 \%)$ & $258(1.6 \%)$ \\
\hline Other & $624(15 \%)$ & $106(11.4 \%)$ & $836(8 \%)$ & $88(11.4 \%)$ & $1654(10.1 \%)$ \\
\hline No complications recorded & $2944(70.7 \%)$ & $744(80 \%)$ & $9027(86.5 \%)$ & $623(80.6 \%)$ & $13338(81.8 \%)$ \\
\hline
\end{tabular}

severe TBI cohort were bronchopneumonia $(4.9 \%)$, in-hospital seizure $(2.9 \%)$, sepsis $(3.1 \%)$ and pleural effusion $(2 \%)$. These were also the most common complications in the cohort as a whole.

\section{Risk-adjusted outcomes at neurosurgical units}

Figure 4 shows a funnel plot $^{12}$ of the risk-adjusted $\mathrm{W}$ scores derived using the Ps $14^{\mathrm{n}}$ model $\left(\mathrm{Ws}^{\mathrm{n}}\right)$ for each unit on the y-axis against a precision (1/SE) based rank on the $\mathrm{x}$-axis. A positive $\mathrm{Ws}^{\mathrm{n}}$ indicates that a site is performing better than the model predicts, a negative value indicates worse performance. The 'funnel' refers to the 2 and 3 SD lines, plotted around the mean Ws that narrow as the precision increases. All units are within the $3 \mathrm{SD}$ lines and most units fall within the $2 \mathrm{SD}$ lines; four units are outside the -2 SD line and two units are above the +2 line. The $\mathrm{Ws}^{\mathrm{n}}$ value for a given site relative to the position of the $\mathrm{SD}$ lines indicates if their performance significantly differs from that of their peers. 


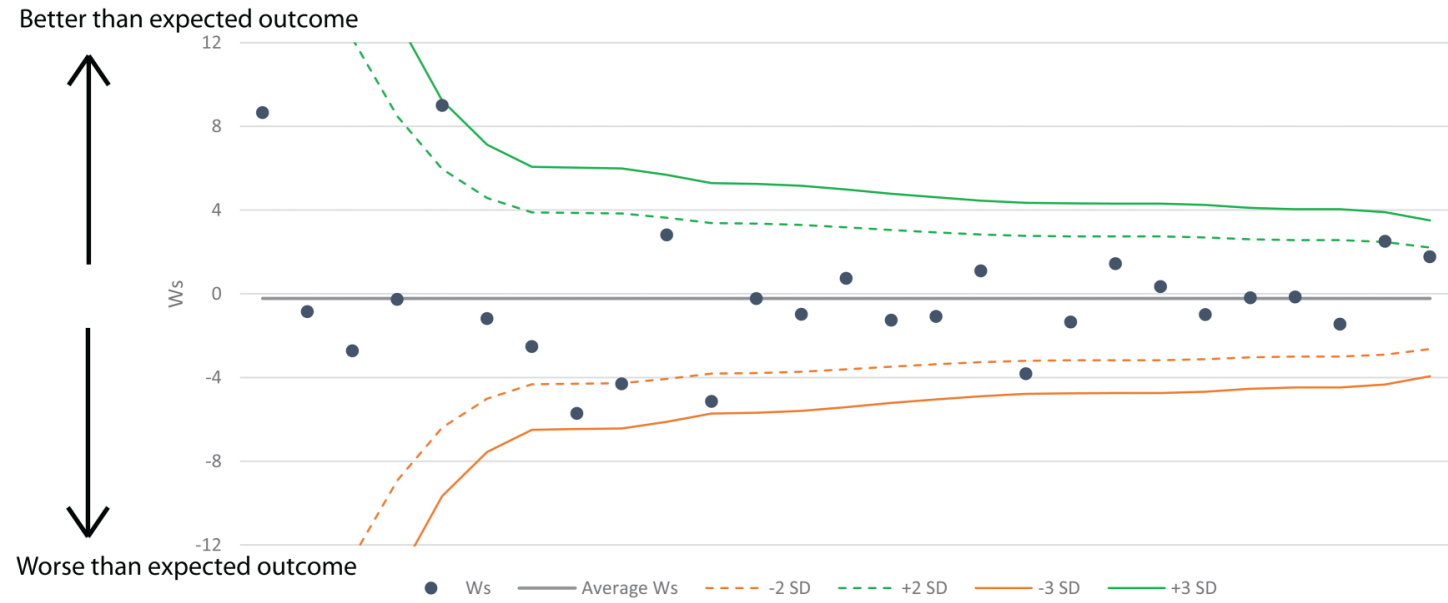

Figure 4 Funnel plot showing the Ws ${ }^{n}$ for neurosurgical units in England and Wales between April 2014 and June 2015. Ws ${ }^{n}$, W scores derived using the Ps14 multivariate logistic regression model.

\section{DISCUSSION}

This audit incorporates prospectively collected data on a large number of patients, including from every neurosurgical unit in England and Wales, and provides the most comprehensive and up to date report of outcomes following TBI in England and Wales.

\section{Demographics and mechanism of injury}

The cohort of patients in the TARN database mimics data from other large TBI databases and the demographics and mechanism of injury closely mirror those from other series of patients with TBI in the developed world. ${ }^{13-15}$ The most common injuries are those in elderly people following trips and falls, while in younger patients, the most common causes are road traffic collisions and assault and these are more likely to present as severe TBI. We have provided a breakdown of delay to transfer to neurosurgical centre and complication rates by 10-year age bracket in the online supplementary information. This demonstrates that despite comparable transfer times between adult groups, there are a smaller number of children aged $0-10$ years transferred within 4 hours $(32 \%)$ as compared with adult age brackets (range $45-61 \%$ ). This does not lead to an increased frequency of complications and we speculate that this is due to specialised transfer team involvement for young children (Children's Acute Transfer Service, CATS). Interestingly, only $6 \%$ of patients with TBI fall into the moderate (GCS 9-13) category calling into question whether the current GCS thresholds for severity accurately reflect the underlying condition: intuitively, one might expect that more severe injuries are increasingly rare. Other epidemiological studies in high-income countries reinforce this pattern of falls as a common aetiology in elderly patients. ${ }^{15}$

\section{Transfer to hospital}

While the majority of patients are transported to hospital by land ambulance, there is an increasing use of helicopter ambulance for those patients with severe TBI. These patients are increasingly being transported directly to major trauma centres (MTCs) as part of the National Health Service (NHS) plan to centralise the management of complex trauma. The choice of mode of transport to hospital and choice of local hospital versus a neurosurgical or MTC is a complex one. Factors such as the physiological stability of a patient on scene and the geography of local emergency services dictates individualisation of decision-making and it is difficult to mandate transport of a group of patients to a given location. A recent publication from the TARN registry ${ }^{16}$ found no association between the duration of the prehospital interval and deteriorating physiological parameters. We did not find a difference in complication rate between these two cohorts (see online supplementary information). There are also challenges with the reliable identification of TBI in the prehospital environment and current strategies suffer from significant undertriage and overtriage rates making secondary transfer into neurosurgery, a necessary pathway for some patients with TBI. ${ }^{17}$ However, in patients with severe TBI, who are likely to survive and require treatment, we would expect transfer to a neurosurgical centre once physiological stability has been achieved. ${ }^{18}$ This is supported by NICE guidance-in our series $84 \%$ of patients with severe TBI received neuroscience care, suggesting reasonable adherence. For mild and moderate TBI, an individual decision is required as to the need and rapidity of transfer to a neurosurgical centre. In a resourcelimited environment however, an efficient use of specialist beds necessitates some degree of triage at local centres before transfer to a specialist centre.

\section{Time to intervention}

The median and upper quartile time to CT is within the 1-hour from ED arrival target defined by the NICE head injury guidelines ${ }^{18}$ for patients at high risk of TBI requiring neurosurgery $(\mathrm{GCS}<13=$ moderate/severe $\mathrm{TBI})$; 
NICE recommends CT brain scan for GCS 15 patients with additional risk factors but not high risk, within 8 hours of injury. Patients with mild TBI with GCS 13-14 on arrival at hospital should have CT within an hour if the GCS does not reach 15 within 2 hours of injury. Sequential ED GCS readings are not well recorded on TARN but table 2 suggests that this NICE recommendation also has reasonable adherence. The Brain Trauma Foundation surgical guidelines ${ }^{19}$ recommend that acute intracranial haemorrhages are treated as quickly as possible in those patients presenting in coma. The evidence for rapid treatment by craniotomy is strongest in those presenting with a fixed, dilated pupil. ${ }^{20}$ In this regard, our data show direct transfer to a neurosurgical centre facilitates more rapid surgery and as such we support current ambulance service trauma triage guidelines that direct primary transportation from scene to a neurosurgical centre for patients with a unilateral fixed, dilated pupil in the context of severe TBI and a patent airway. ${ }^{17}$ Consideration should also be given to establishing guidelines for direct transfer of other patients with TBI from the scene to neurosurgical units, notwithstanding the difficulty in accurate identification of patients in the prehospital setting, and refining referral mechanisms from district hospitals/trauma units to MTCs with neurosurgical capability. Any guidelines must reflect the low requirement for craniotomy and ICP monitoring in mild $(3.1 \%$ and $0.7 \%)$ and moderate $(2.7 \%$ and $2.1 \%)$ TBI, such that in the majority of these patients expedited transfer to a neurosurgical centre may be unnecessary.

\section{Complications and risk-adjusted outcomes at neurosurgical units}

Patients with TBI are susceptible to a wide range of complications as evidenced by the reported complications. Respiratory complications predominate as would be expected in critically ill patients with a reduced conscious state or those in an intensive care environment. The analysis shows that five units lie outside of the $2 \mathrm{SD}$ control limits; however, they and all other units are within the $3 \mathrm{SD}$ limits. A single centre is close to the positive $3 \mathrm{SD}$ limit, but this is one of the units with lower precision where we expect to see larger variation from the mean. As such these data suggest that there are no outlying units in terms of risk-adjusted mortality in neurosurgical care for patients suffering from TBI in England and Wales. Further studies are required to address the quality of survival in terms of outcome beyond mortality. On the basis of the funnel plot, it appears that there is a slight excess of units falling below the expected standardised mortality ratio (worse than expected outcome). This is most likely due to the expected (average) value being skewed upwards by the two centres with low precision and very high $\mathrm{Ws}^{\mathrm{n}}$ scores. In addition, a significant proportion of the centres below the expected value are those with lower precision, the higher precision units on the right side of the plot are more evenly balanced.

\section{Study limitations}

Although this audit is comprehensive, there are certain limitations to using aggregate data of this type. First, as with many studies that use GCS, we have used a composite score rather than the individual components, despite each component of the GCS being on a categorical scale. This is partly addressed by the validation of this approach by the IMPACT model. ${ }^{10}{ }^{21}$ Second, there is some variability in the reporting of GCS, such that 'first' GCS is sometimes used interchangeably with 'postresuscitation' GCS. $^{21}$ Third, we have not addressed the decision-making with regard to transfer of patients from peripheral to neurosurgical centres, and the possibility of regional variation. This could potentially have an effect on TBI survival rates in specialist centres if there is a variation in transfer criteria, particularly for older patients who may have poorer prognosis. ${ }^{22}$ Finally, there is some variability in patient recruitment into the TARN database, over the time period of the study neuroscience centres recruited almost $100 \%$ of relevant patients, outside of these hospitals, however the average is roughly $65 \%$. Nevertheless, we hope by compiling data on more than 15000 patients, we are able to provide robust data on TBI management in England and Wales.

\section{CONCLUSION}

This report provides the first England and Wales audit of its type with a large number of patients that is commensurate with the largest cohorts of patients currently published in TBI, namely the CRASH and IMPACT studies. This provides a robust baseline for further comparisons of outcomes in a transparent and reproducible fashion. The data we present confirm that England and Wales trauma management broadly meets the NICE guidelines and achieves a consistent standard across all regions and neurosurgical units. The NICE guidelines are broad and rightly err on the side of caution in the necessity for CT imaging and discussion with specialist centres. ${ }^{18}$ Specifically, they are for the management of head injury, rather than traumatic brain injury, and the recommendations address CT imaging and appropriate transfer to neurosurgical centres, rather than ICP monitoring and the need for craniotomy, although this is a possibility in the future. The need for these guidelines to be used in a range of ED settings necessitates this approach, although data presented here highlight that neurosurgical intervention is rarely required for those presenting with mild or moderate TBI. The increasing need for public engagement with regard to surgical outcomes, and the related political imperative to provide this within the NHS will become the status quo.

\section{Author affiliations}

${ }^{1}$ Trauma Audit and Research Network, Manchester Medical Academic Health Sciences Centre, Institute of Population Health, University of Manchester, Salford Royal Hospital, Salford, UK

${ }^{2}$ Division of Neurosurgery, Department of Clinical Neuroscience, University of Cambridge, Cambridge, UK 
${ }^{3}$ Department of Neurosurgery, Addenbrooke's Hospital, Cambridge, UK ${ }^{4}$ Centre for Urgent and Emergency Care Research (CURE), Health Services Research Section, School of Health and Related Research (ScHARR), University of Sheffield, Sheffield, UK

Contributors FL and PJH conceived of the study and have provided detailed review of the results. $\mathrm{AH}$ and $\mathrm{TL}$ have written the manuscript. TL, OB and MW have carried out the statistical analysis.

Funding AH is supported by the University of Cambridge, UK and Medical Research Council/Royal College of Surgeons of England Clinical Research Training Fellowship (grant number G0802251). PJH is supported by National Institute for Health Research Professorship, Academy of Medical Sciences/ Health Foundation Senior Surgical Scientist Fellowship and the National Institute for Health Research Biomedical Research Centre, Cambridge.

Competing interests None declared.

Provenance and peer review Not commissioned; externally peer reviewed.

Data sharing statement No additional data are available.

Open Access This is an Open Access article distributed in accordance with the terms of the Creative Commons Attribution (CC BY 4.0) license, which permits others to distribute, remix, adapt and build upon this work, for commercial use, provided the original work is properly cited. See: http:// creativecommons.org/licenses/by/4.0/

\section{REFERENCES}

1. National Institute for Health and Clinical Excellence: Guidance. Head injury: triage, assessment, investigation and early management of head injury in children, young people and adults. London: National Institute for Health and Care Excellence (UK), 2014.

2. ATLS Subcommittee; American College of Surgeons' Committee on Trauma; International ATLS working group. Advanced Trauma Life Support (ATLS): the ninth edition. J Trauma Acute Care Surg 2013;74:1363-6.

3. Patel HC, Menon DK, Tebbs S, et al. Specialist neurocritical care and outcome from head injury. Intensive Care Med 2002;28:547-53.

4. Stein SC, Georgoff P, Meghan S, et al. Relationship of aggressive monitoring and treatment to improved outcomes in severe traumatic brain injury. J Neurosurg 2010;112:1105-12.

5. Perel $\mathrm{P}$, Arango M, Clayton $\mathrm{T}$, et al. MRC CRASH Trial Collaborators. Predicting outcome after traumatic brain injury: practical prognostic models based on large cohort of international patients. BMJ 2008;336:425-9.
6. Murray GD, Butcher I, McHugh GS, et al. Multivariable prognostic analysis in traumatic brain injury: results from the IMPACT study. J Neurotrauma 2007;24:329-37.

7. Neurosurgical National Audit Programme. 2014. http://www.hed.nhs. uk/SBNS/

8. Abbreviated Injury Scale (AIS). 2014. http://www.aaam.org/about-ais. htm

9. Bouamra O, Jacques R, Edwards $A$, et al. Prediction modelling for trauma using comorbidity and 'true'30-day outcome. Emerg Med J 2015;32:933-8.

10. Marmarou A, Lu J, Butcher I, et al. Prognostic value of the Glasgow Coma Scale and pupil reactivity in traumatic brain injury assessed pre-hospital and on enrollment: an IMPACT analysis. J Neurotrauma 2007;24:270-80.

11. Hollis S, Yates D, Woodford M, et al. Standardized comparison of performance indicators in trauma: a new approach to case-mix variation. J Trauma 1995;38:763-6.

12. Spiegelhalter DJ. Funnel plots for comparing institutional performance. Stat Med 2005;24:1185-202.

13. Peeters W, van den Brande R, Polinder S, et al. Epidemiology of traumatic brain injury in Europe. Acta Neurochir (Wien) 2015;157:1683-96.

14. Maas Al, Murray GD, Roozenbeek B, et al. Advancing care for traumatic brain injury: findings from the IMPACT studies and perspectives on future research. Lancet Neurol 2013;12:1200-10.

15. Walder B, Haller G, Rebetez MM, et al. Severe traumatic brain injury in a high-income country: an epidemiological study. J Neurotrauma 2013;30:1934-42.

16. Fuller G, Lawrence T, Woodford M, et al. Emergency medical services interval and mortality in significant head injury: a retrospective cohort study. Eur J Emerg Med 2015;22:42-8.

17. Lecky F, Russell W, Fuller G, et al. The Head Injury Transportation Straight to Neurosurgery (HITS-NS) randomised trial: a feasibility study. Health Technol Assess 2016;20:1-198.

18. NICE. Triage, assessment, investigation and early management of head injury in children, young people and adults. 2014. http://www. nice.org.uk/guidance/cg176/evidence

19. Guidelines for the management of severe traumatic brain injury. 2007. https://www.braintrauma.org/

20. Clusmann $\mathrm{H}$, Schaller $\mathrm{C}$, Schramm J. Fixed and dilated pupils after trauma, stroke, and previous intracranial surgery: management and outcome. J Neurol Neurosurg Psychiatr 2001;71:175-81.

21. Reith FC, Van den Brande R, Synnot A, et al. The reliability of the Glasgow Coma Scale: a systematic review. Intensive Care Med 2016;42:3-15.

22. Kirkman MA, Jenks T, Bouamra O, et al. Increased mortality associated with cerebral contusions following trauma in the elderly: bad patients or bad management? J Neurotrauma 2013;30:1385-90. 